\title{
An exploration of the protective mechanism of esmolol on the heart of septic rats based on the beta-adrenergic signaling pathway.
}

\section{Pan Li}

Lanzhou University Second Hospital

Jingzhuo Liu

Lanzhou University Second Hospital

\section{Xintong Zhang}

Lanzhou University Second Hospital

\section{Lingling Zhen}

Lanzhou University Second Hospital

\section{Yingzhen Wang}

Lanzhou University Second Hospital

\section{Xiaorong Dong}

Lanzhou University Second Hospital

\section{Bei Zhang}

Lanzhou University Second Hospital

\section{Shuhang Luo}

Sun Yat-sen University Zhongshan School of Medicine

\section{Li Ma ( $\nabla$ ery_mali@lzu.edu.cn )}

Lanzhou University Second Hospital

\section{Research}

Keywords: Sepsis; Myocardial dysfunction, Esmolol; $\beta$-adrenergic signaling pathway; Receptor desensitization

Posted Date: March 5th, 2020

DOl: https://doi.org/10.21203/rs.3.rs-16199/v1

License: (c) (1) This work is licensed under a Creative Commons Attribution 4.0 International License.

Read Full License 


\section{Abstract}

Background Cardiac dysfunction caused by sepsis is a serious challenge for ICU doctors. Previous studies showed that the beta adrenergic receptor ( $\beta$ - AR) signaling pathway system can cause abnormal heart function. Studies have also shown that esmolol, a type of beta blocker, participates in the beta receptor desensitization that occurs. This study intends to further explore the specific mechanism of esmolol in the protection of cardiac function.

Method In this study, rats were randomly divided into three groups: Sham (sham operation group), CLP (cecal ligations and function group) and ES (esmolol group). The three groups were divided into two subgroups: A and B. Group A was used to observe the number of survival days. Group B was divided into 6 hours, 12 hours and 24 hours according to the time after the operation to take samples, and the rats were sacrificed after the corresponding intervention. The inflammatory factors and the factor of $\beta$ receptor pathway were measured by different methods. And the hypothesis was verified by statistical methods.

Results ELISA tests demonstrated that the levels of inflammatory factors CA,TNF-a, and IL-6, as well as G protein pathway factors AC, CAMP and PKA in the CLP group were significantly higher than those in the Sham group, while those factors in the ES group were significantly lower than those in the CLP group, cardiac function factor $\mathrm{CTnl}$ is the opposite. Western blotting showed that, compared with the CLP group, the inflammatory proteins MYD88 and NF-KB in the myocardial tissue of the ES group were significantly decreased, while the levels of the beta receptor pathway proteins $\beta 1$-AR, $\beta 2$-AR, $\beta$-arrestin- 1 and $\beta$-arrestin2 were significantly increased, and the result of $\beta$-ARK was the opposite. Pathological results and immunofluorescence results indicated that the degree of myocardial cell injury in the ES group was significantly improved compared with that in the CLP group.

Conclusion Esmolol has a protective effect on the heart by selectively preventing the pathologic signaling pathway mediated by overactivation of beta receptors while retaining the normal signaling pathway.

\section{Introduction}

Sepsis is a life-threatening organ dysfunction caused by disordered host response to infection, which is manifested as systemic inflammatory response and host autoimmune injury[1]. At the same time, it is complicated with multiple organ dysfunction syndrome, most of which are circulatory, immune and metabolic dysfunctions. A previous study showed that sepsis not only causes great damage to the body, but also makes the social burden significantly increased[2][3]. It also increases the hospitalization rate and mortality of patients year by year. More than 52, 000 people die of sepsis each year[4]. A recent study in the United States has shown that $12 \%$ of sepsis cases are fully preventable[5]. The mechanism of sepsis has also been an interest of researchers for a long time. Sepsis is now considered to be an alternate process of immunosuppression and hyperimmunity[6][7]. 
Past research has shown that G-protein coupled receptors (GPCRs) can be combined with various bioactive molecules, including extracellular signals from hormones, neurotransmitters, chemical hormones and other environmental stimuli, and that GPCRs induce cellular gene transcription changes via different signaling pathways, participating in the regulation of almost all of the physiological processes. A study of 601 subjects showed that catecholamines are an important predictor of cardiovascular mortality, MI and stroke[8].The body, in the case of any damage, poisoning or other cause of injury or stress state, releases a large amount of catecholamines, resulting in systemic hypercatabolism, hyperdynamic circulation and abnormal magnification of inflammatory response, leading to multiple organ dysfunction. Over-activated catecholamines can induce calcium overload and can cause changes in signal pathway transduction. From activating the $\beta$-AR-Gs-AC-cAMP-PKA pathway to activating the $\beta$-AR-Gs-Ca2-CaMKII pathway, cardiomyocyte apoptosis is promoted and leads to sepsisinduced myocardial dysfunction (SIMD). SIMD is one of the most important causes of the poor prognosis of sepsis.

$\beta$-arrestin is a kind of header protein and signal transduction regulatory protein found in the process of purification of $\beta$ adrenergic receptor kinase 1( $\beta-A R K 1)[9][10][11]$. It has many physiological functions. It has been proven that it can not only participate in the occurrence of $\beta$ receptor desensitization in septic cardiac dysfunction, but also activates the ERK signal pathway by the activation of epidermis factor translocation, thus inhibiting the apoptosis of cardiomyocytes induced by continuous activation of $\beta$ receptor and giving full play to the protective effect of the heart. Previous studies show that $\beta$-arrestin and $\beta$-ARK1 are two important factors involved in $\beta$-receptor desensitization[12]. When the agonist binds to $\beta$ AR, the agonist-activated $\beta$-AR binds to $\beta$-ARK1 and undergoes phosphorylation, which causes the conformational change of $\beta$-arrestin and the increase of affinity of $\beta$-arrestin to $\beta$-AR, this in turn inhibits the interaction between GPCRs and G protein, thus hindering the signal transduction of GPCRs[13][14]. $\beta$ arrestin is also directly involved in the degradation of second messenger CAMP, and the inhibition of downstream cAMP-dependent mitogen-activated protein kinase(MAPK)activation, thus promoting desensitization. A large number of studies have shown that a variety of factors are alone or jointly involved in $\beta$-arrestin induced $\beta$ receptor internalization, that is, the $\beta$ receptor on the surface of the cell membrane enters the membrane, which weakens the signal stimulation and leads to the desensitization of $\beta$ receptor[15][16]. The endocytized receptor can be re-released into the cell membrane cycle to continue to receive stimulation, but also can be degraded by the related enzymes, being made completely defunct[17].

Esmolol, as an ultra-short-acting selective $\beta 1$ receptor blocker, has been confirmed, at home and abroad, to have cardiac protective effect, and to reduce the hospitalization rate and mortality of septic patients with cardiac dysfunction[18][19]. The results of animal experiments also confirmed that it could reverse left ventricular hypertrophy[20]. Human randomized controlled trials and other related studies have also shown that esmolol can improve the survival rate of septic patients and slow down the heart rate but does not affect the oxygen index[21]. It inhibits the $\beta 1$ receptor by competing for catecholamine binding sites on the surface of cardiomyocytes and suppressing the hypermetabolism of catecholamine overactivation in the whole body[22]. The purpose of this study was to explore the specific mechanism of 
esmolol in improving cardiac function from the organ, tissue and cellular levels, to find new targets and to provide theoretical support for clinical reduction of sepsis mortality and improvement of prognosis.

\section{Material And Methods}

96 SPF male rats (250-300 g) were provided by the Experimental Animal Center of Lanzhou University School of Basic Medicine, animal license number: SCXK (Gan). The experiment was approved by the Experimental Animal Ethics Committee of the Second Affiliated Hospital of Lanzhou University.

\section{Animals}

Before examination, the rats were fed and watered freely in the laboratory at room temperature of $22{ }^{\circ} \mathrm{C}$ to $26{ }^{\circ} \mathrm{C}$ and humidity of $40-60 \%$. The rats were fed adaptively for 1 week after 12 hours of light and dark cycles. The experimental animals were randomly divided into 3 groups $(n=72)$, namely, Sham group, CLP group and ES group, and 8 rats from each group were randomly selected for observation of the survival condition at 7 days. The remaining 24 rats were randomly divided into 6,12 and $24 \mathrm{~h}$ subgroups according to the experimental time, with 8 in each subgroup.

\section{Experimental Animal Model}

Rats were prepared by receiving no food for 12 hours (water given ad lib) and then $2 \%$ sodium pentobarpital was injected into the abdomen. The rats' limbs were fixed in supine position, the abdomen was prepped and draped. An approximately $2 \mathrm{~cm}$ vertical incision was made in the abdomen and the abdominal cavity was entered layer by layer. The mesentery and cecum were mobilized and the distal $1 / 3$ of the cecal artery was ligated with 4.0 silk suture. In the CLP and ES groups, the end of the cecum was perforated twice with a 20 gauge sterile needle and a small amount of intestinal contents extruded from the cecum into the abdominal cavity. In the Sham group, no artery ligation or perforation of the cecum was performed. The abdominal cavity was closed in layers. Animals received $20 \mathrm{~mL} / \mathrm{kg}$ of normal saline as fluid resuscitation post operatively.

The successful establishment of the model was marked by mental malaise, vertical hair, and secretion in the corners of the eyes. Laparotomy at the corresponding time point revealed a large amount of ascitic fluid and ligated blackened cecum adhesions, and foul smell.

\section{Internal jugular vein catheterization in rats and administration of drugs in experimental animals}

The skin of the left neck of the rats was prepped and draped then an incision was made from the upper left to lower right. The distal portion of the internal jugular vein was ligated, a "V" shaped incision was made using ophthalmologic scissors, and the jugular vein was cannulated with a catheter primed with heparin sodium $(25 \mathrm{U} / \mathrm{mL})$. Blood return was used to indicate successful catheter placement. Once the catheter was secured to the distal ligature, $0.5 \mathrm{~mL}$ of heparin sodium was instilled. The incision was covered with wet gauze. In the sham group, saline infusion was continued for 6 hours. The ES group had 
continuous infusion of esmolol at a dose of $15 \mathrm{mg} / \mathrm{kg} / \mathrm{hr}(1 \mathrm{~mL} / \mathrm{h})$, for a total of 6 hours. The animal's symptoms and heart rate were monitored continuously during esmolol infusion and esmolol dose was adjusted over time based on observations. Post-infusion the catheters were removed, blood vessels ligated and incision sutured closed.

\section{Experimental Protocols}

The rats in each group were killed at the corresponding time point $(6,12$, and $24 \mathrm{~h})$. After thoracotomy, $4 \mathrm{~mL}$ blood samples were collected from the heart, at room temperature for $10 \mathrm{~min}$, and then centrifuged at $2000 \mathrm{r} / \mathrm{min}$ for $5 \mathrm{~min}$. The related biochemical indices were collected and stored at low temperature after loading. The heart was removed and the myocardial tissue was preserved in $-80^{\circ} \mathrm{C}$ cryopreservation box, and the homogenate and related biochemical indexes were to be detected.

\section{Detection of serum CA, TNF- $a$, IL-6, cTnl, AC, cAMP, and PKA levels by ELISA in rats}

Using ELISA kit (Jianglai, Shanghai, China) of CA, TNF-a, IL-6, cTnl ,AC, CAMP and PKA were used and operated in strict accordance with the instructions, and the absorbance was measured at $450 \mathrm{~nm}$ by using a microplate reader, and the standard curve was drawn to calculate the levels of the above factors.

Western blot was used to detect the content of the inflammatory protein (MYD88, NF-KB) and the receptor-pathway proteins ( $\beta 1-A R, \beta 2-A R, \beta A R K 1, \beta a r r e s t t i n-1, \beta$-arresttin-2) in the myocardium.

An appropriate amount of myocardial tissue was taken and centrifuged after tissue lysis, and the supernatant was taken. We used the BCA quantitative method, denaturing the protein and running the gel, transferring the mold and blocking with $5 \%$ skim milk at room temperature for $1.5 \mathrm{~h}$. Then NF-KB (1: 500), MYD88 (1: 1000), $\beta 1-A R ~(1: 1000), \beta 2$-AR (1: 1000), $\beta A R K ~(1: 1000), \beta$-arrestin1 (1: 500), $\beta$-arrestin2 (1: $1000)$, $\beta$-tublin (1:20000), GAPDH (1: 10000) were diluted respectively and incubated in a refrigerator at 4 ${ }^{\circ} \mathrm{C}$ overnight. The next day, the film was washed with TBST at room temperature 3 times, 10 min each time. As in the previous method, the secondary antibody (1:2 000) was incubated at room temperature for $1 \mathrm{~h}$, and developed with ECL working fluid after three washings. The signal was obtained by radiography equipment. Finally, Image J software was used for image analysis and processing, and the gray values of target protein and internal parameters were obtained. The relative content of the target protein is equal to the grey value of the target protein divided by the grey value of the internal parameters multiplied by $100 \%$.

\section{The pathological observation of myocardial tissue}


Myocardial tissue was washed with saline 3 times, fixed with $10 \%$ neutral formaldehyde buffer, dehydrated, embedded, sliced, roasted, stained with paraffin and hematoxylin-E red, and the morphological changes were observed under light microscopy.

\section{Myocardial cell apoptosis under fluorescence microscopy}

Frozen sections were removed from the refrigerator and rewarmed. They were air dried, fixed, decolorized, washed, repaired and membranes broken up. Reagent 1 (TdT) and reagent 2 (dUTP) were added. The appropriate amount of reagent from the TUNEL kit(BestBio,Shanghai,China) was determined according to the number of slices and tissue size. Reagents 1 and 2 were mixed at $2 \mathrm{~m} 29 \mathrm{~s}$ to block endogenous peroxidase. Reagent 3 (converter-POD) was added to cover the tissue and develop DAB color. Sections were rinsed with tap water to stop the color development.

\section{Survival rate analysis}

The rats in the Sham group, CLP group and ES group were given normal diet after 6 hours, and the survival of each group was observed.

\section{Data analysis method}

Statistical analyses were done using the software Statistical Product for Social Sciences (SPSS; version 16.0, Chicago, IL, USA) and data plotted using GraphPad Prism 5.0 Software (San Diego, CA, USA). Differences among groups were tested using a one-way analysis of variance. Data are expressed as mean \pm SEM. A p-value $<0.05$ was considered statistically significant.

\section{Results}

\section{Esmolol can reduce the inflammation of myocardial tissue in rats by inhibiting MYD88/ NF-KB pathway}

As shown in Figs. 1, 2, 3,5,6 and 7,the results of ELISA showed that the levels of serum CA,TNFa,AC,CAMP,PKA and IL- 6 in the CLP group and the ES group were significantly higher than those in the Sham group at $6 \mathrm{~h}, 12 \mathrm{~h}$ and $24 \mathrm{~h}$ after operation. The levels of those factors in the ES group were significantly lower than those in the CLP group at each time point $(P<0.05)$, while cTnl showed the opposite (Fig. 4). From the Western blot results of Figs. 8 and 9,we can see that the expression levels of MYD88 and NF-KB in the ES group were significantly lower than those in the CLP group at 6, 12, and 24 hours after surgery. NF-KB protein expression was significantly higher than that in Sham group, and the expression of MYD88 and NF-KB protein in the ES group was significantly lower than that in the CLP group at each time point $(P<0.05)$. 


\section{Esmolol can improve $\beta$-AR desensitization and signal transduction in cardiomyocytes by upregulating $\beta 1-A R, \beta 2-$ $A R, \beta$-arrestin-1, and $\beta$-arrestin-2 protein expression}

The results of the Western blot show that the expression of $\beta 1-A R, \beta 2-A R$, $\beta$-arrestin-1, and $\beta$-arrestin-2 proteins in the CLP group and the ES group was significantly higher than that in the Sham group at $6 \mathrm{~h}$, $12 \mathrm{~h}$, and $24 \mathrm{~h}$ after operation. The expression of those proteins in the ES group was significantly lower than that in the CLP group at all time points $(P<0.05)$. When compared longitudinally in chronological order, taking the CLP group as an example, we found that the expression of the $\beta$ receptor pathway proteins in cardiomyocytes was the least at $12 \mathrm{~h}$, followed by $6 \mathrm{~h}$, and the expression of $24 \mathrm{~h}$ increased slightly, which was similar to that in the Sham group and the ES group. And $\beta A R K$ is completely different from the above proteins. This can be seen in Figs. 10, 11, 12,13 and 14.

The results of pathological tissue show that esmolol could reduce the degree of cardiac function damage in sepsis.

As can be seen from the Fig. 15(20x), the number of myocardial fibers in the CLP group decreased, the cells increased, and the transverse striations were blurred, broken, and irregular, with more inflammatory cells and a greater degree of tissue damage. The conditions in the ES group were slightly improved, and the Sham group had the least damage.

The results of the Tunel staining suggest that esmolol can reduce the number of apoptotic cells and improve the myocardial injury.

The results of Tunel staining are shown in Fig. 16A(20x). We used the ImageJ software to quantitatively analyze the staining results, and the results are shown in Fig. 16B. Compared with the sham group, the apoptotic area of the ES group and the CLP group were significantly increased. Compared with the CLP group, all subgroups of the ES group showed apoptotic area that is significantly reduced, suggesting esmolol can protect the myocardium to some extent.

\section{Esmolol can improve the survival rate of rats}

We used the log-rank test for survival analysis to 15 days to compare the rat survival curve of the Sham group, the CLP group, and the ES group. Using survival analysis at 15 days, the CLP group had the worst outcomes, and the Sham group had more rats survive ( $<<0.01$, see Fig. 17).

\section{Discussion}

Sepsis is a syndrome of immune imbalance involving multiple organs, which can lead to abnormalities in various organs of the body, among which the heart is the most severely damaged. Cardiac dysfunction induced by sepsis is the main cause of poor prognosis in patients with sepsis. The treatment of heart 
dysfunction has also become a major effort of clinicians. As a beta blocker with super-short effect, esmolol has been proven to have a protective effect on the heart many times, and is widely used in clinical practice. In this experiment, samples were obtained through the classical modeling method and drug intervention method, and relevant factors were detected, and the expected results were finally obtained.

It is a well known technique to induce sepsis in the rat model by caecal ligation and puncture. It is also quite reasonable to apply it in this study. ELISA and Western Blot show that after CLP intervention, inflammatory cytokines CA, TNF-a, IL-6, MyD88 and NF-KB were significantly increased compared with the sham group, similar to a septic patient's clinical pathological process of inflammatory activation. In the use of esmolol after the intervention, the content of the above inflammatory factors decreased, which was statistically significant. This indicates that esmolol has the effect of inhibiting the inflammatory response during sepsis, and can reduce the damage of inflammation to the body in the early stage. As for the cardiac function factor cTnl, compared with the CLP group, esmolol can increase the content of cTnl and improve the cardiac function of patients to a certain extent.

Then,we studied the improvement of the beta receptor pathway with esmolol. As mentioned earlier in this article, beta receptors have positive inotropic effects that maintain the general physiological function of the heart by exciting GPCRs, that is $\beta-A R-G s-A C-c A M P-P K A$ pathway. However, when sepsis occurs, the early "sympathetic storm" causes the beta receptors to be continuously excited by catecholamines, and the GPCRs are transformed into the $\beta-A R-G s-\mathrm{Ca}^{2+} \square \mathrm{CaMKII}$ pathway, which triggers apoptosis and cardiac dysfunction. It can be seen from ELISA results that, compared with the Sham group, the levels of G protein pathway factors AC, CAMP and PKA in the CLP group are significantly increased, while the levels of these factors are decreased in the ES group. This suggests that when sepsis occurred, esmolol, as beta blockers, can compete for catecholamine binding sites and inhibit the early onset of the "sympathetic storm" of sepsis. In other words, it can inhibit the pathologic apoptotic pathway mediated by catecholamines, thus playing a protective role on the heart.

Previous data have shown that $\beta$-arrestin is involved in the process of reducing the incidence of left heart failure in patients and improving the cardiac function of patients with myocardial fibrosis to a certain extent[23][24]. There are also studies that show that $\beta$-arrestin can protect the myocardium in two ways. First, it is involved in the degradation, internalization, and circulation of $\beta$-AR mediated by $G$ protein of the classical pathway, and it inhibits the apoptotic pathway induced by overactivated beta receptors in the early stage of sepsis. As previously stated, this desensitization cannot occur without both beta-arrestin and beta-ARK. Secondly, it can also act as a signaling molecule to participate in the beta receptor pathway, that is, it can bind to the beta receptor to activate the extracellular signal-regulated kinase (ERK)., inhibiting the proliferation and apoptosis of cardiomyocytes, thereby improving cardiac function. In this experiment, western blot showed that compared with the CLP group, the levels of $\beta 1-A R, \beta 2-A R, \beta-$ arrestin-1 and $\beta$-arrestin-2 in the myocardial tissue of the ES group were significantly increased. It is suggested that in the early stage of sepsis, esmolol can activate the $\beta$-arrestin pathway to trigger the desensitization of beta receptors and prevent their over-activation thereby increasing the expression level 
of beta receptors and ultimately reducing the occurrence of cardiac dysfunction. Previous studies have shown that $\beta A R K$ is involved in the occurrence of myocyte hypertrophy[25], while the results of our experiment are just the opposite of the previous protein expression, indicating that esmolol can play a protective role in myocytes by reducing the expression of $\beta A R K$.

In addition, our pathological results showed that, among the myocardial cell injuries in each time period, the sham group was the least severe, followed by the ES group, and the CLP group was the most severe. It also visually demonstrated the cardiomyocyte protective effect of esmolol. We analyzed the apoptotic area of cardiomyocytes under electron fluorescence microscopy, and the immunofluorescence results also showed the same conclusion.

Finally, as shown in the figure of survival rate analysis, esmolol did make a significant contribution to the improvement of rat mortality and indirectly indicating a protective function in cardiomyocytes.

In general, from the perspective of desensitization of the $\beta$ receptor in the pathogenesis of SIMD, we have explored its mechanism from the whole, tissue, and cellular levels. Taking $\beta A R K 1, \beta$-arrestin- 1 and $\beta$ arrestin-2 as the key observational indicators, we have provided a new target for clinical treatment of SIMD, providing a new theoretical basis for esmolol to improve SIMD, and providing theoretical support for clinical reduction of sepsis mortality and improvement of prognosis. All of these have not been or only rarely been previously studied.

Of course, there are still many deficiencies in this study. For example, the literature selected in this study is limited, and the data on the relationship between esmolol and beta receptor pathway is relatively insufficient. The theoretical aspect is not yet systematic and mature, it is still in the preliminary research stage. The experimental scheme is not perfect, and the sample size is limited. We also fail to face the above research from the genetic level. These defects will become our future research direction.

\section{Conclusion}

In summary, sepsis is a very complex pathophysiological process. Esmolol, as a kind of beta blocker, plays a huge role in the improvement of heart function during sepsis. The mechanism may be related to the fact that esmolol blocks the pathological signaling pathway induced by over-activation of beta receptors in sepsis. At the same time, it also preserves the physiological signaling pathway mediated by the beta-arrestin pathway, as well as improves the inflammatory response of myocardial cells and plays a role in the protection of the myocardial cells from multiple aspects. In the future, we should continue to explore the specific mechanism, providing new targets for the treatment of sepsis and new ideas for the better application of esmolol in clinical practice.

\section{Declarations}

\section{Acknowlegements}


Not applicable

\section{Funding}

This work was supported by the Project fund of the Lanzhou University of Second Hospital(NO.CY-2018MS03).

\section{Availability of data and materials}

All data generated or analyzed during this study are included in this published article.

\section{Author information}

\section{1)Affiliations}

Department of Critical Care Medicine, Lanzhou University Second Hospital,No. 82, Cuiyingmen,Chengguan District, Lanzhou City, Gansu Province, China:

Pan Li,Jing-zhuo Liu,Xin-tong Zhang,Ling-ling Zhen,Ying- Zhen Wang,Xiao-rong Dong,Bei Zhang

Sun Yat-sen University Zhongshan School of Medicine No.74 Zhongshan 2nd Road, Guangzhou, China:

Shu-hang Luo

\section{2)Contributions}

PL, JZL and XTZ participated in data analysis and manuscript drafting, and LLZ contributed to the experimental design, YZW and XRD participated in animal modeling process and specimen acquisition, BZ and SHL participated in specimen detection and data processing. LM participated in manuscript revision for critical intellectual content, and the observation of the whole research process.

\section{Corresponding Author}

Li Ma, MD PhD, E-mail: ery_mali@lzu.edu.cn,Department of Critical Care Medicine, Lanzhou University Second Hospital,No. 82, Cuiyingmen,Chengguan District, Lanzhou City, Gansu Province, China.

\section{Ethics declarations}

The experiment was approved by the Experimental Animal Ethics Committee of the Second Affiliated Hospital of Lanzhou University.

\section{Consent for publication}

Not applicable.

\section{Competing interests}


The authors declare that they have no competing interests.

\section{References}

1. Singer M, Deutschman C S, Seymour C W, et al. The third international consensus definitions for sepsis and septic shock (Sepsis-3)[J]. Jama, 2016, 315(8): 801-810.

2. Zimmerman J E, Kramer A A, Knaus W A. Changes in hospital mortality for United States intensive care unit admissions from 1988 to 2012[J]. Critical care, 2013, 17(2): R81.

3. Mayr F B, Yende S, Angus D C. Epidemiology of severe sepsis[J]. Virulence, 2014, 5(1): 4-11.

4. Singer M, Inada-Kim M, Shankar-Hari M. Sepsis hysteria: excess hype and unrealistic expectations[J]. The Lancet, 2019, 394(10208): 1513-1514.

5. Rhee C, Jones T M, Hamad Y, et al. Prevalence, underlying causes, and preventability of sepsisassociated mortality in US acute care hospitals[J]. JAMA network open, 2019, 2(2): e187571e187571.

6. Hotchkiss R S, Monneret G, Payen D. Sepsis-induced immunosuppression: from cellular dysfunctions to immunotherapy[J]. Nature Reviews Immunology, 2013, 13(12): 862.

7. van der Poll T, van de Veerdonk F L, Scicluna B P, et al. The immunopathology of sepsis and potential therapeutic targets[J]. Nature Reviews Immunology, 2017, 17(7): 407.

8. Peng $Y X$, Shan J, Qi X Y, et al. THE CATECHOLAMINE- $\beta$-ADRENORECEPTOR-cAMP SYSTEM AND PREDICTION OF CARDIOVASCULAR EVENTS IN HYPERTENSION[J]. Clinical and experimental pharmacology and physiology, 2006, 33(3): 227-231.

9. Wang $Y$, Jin L, Song Y, et al. $\beta$-arrestin 2 mediates cardiac ischemia-reperfusion injury via inhibiting GPCR-independent cell survival signalling[J]. Cardiovascular research, 2017, 113(13): 1615-1626.

10. Zhu L, Rossi M, Cui Y, et al. Hepatic $\beta$-arrestin 2 is essential for maintaining euglycemia[J]. The Journal of clinical investigation, 2017, 127(8): 2941-2945.

11. Zhu L, Almaca J, Dadi P K, et al. $\beta$-arrestin- 2 is an essential regulator of pancreatic $\beta$-cell function under physiological and pathophysiological conditions[J]. Nature communications, 2017, 8: 14295.

12. Shukla A K, Xiao K, Lefkowitz R J. Emerging paradigms of $\beta$-arrestin-dependent seven transmembrane receptor signaling[J]. Trends in biochemical sciences, 2011, 36(9): 457-469.

13. Saucerman J J, McCULLOCH A D. Cardiac $\beta$-Adrenergic Signaling: From Subcellular Microdomains to Heart Failure[J]. Annals of the New York Academy of Sciences, 2006, 1080(1): 348-361.

14. Lynch M J, Baillie G S, Mohamed A, et al. RNA silencing identifies PDE4D5 as the functionally relevant CAMP phosphodiesterase interacting with $\beta$ arrestin to control the protein kinase A/AKAP79mediated switching of the $\beta 2$-adrenergic receptor to activation of ERK in HEK293B2 cells[J]. Journal of Biological Chemistry, 2005, 280(39): 33178-33189.

15. Luttrell L M. Regulators of GPCR Activity[M]//The G Protein-Coupled Receptors Handbook. Humana Press, 2005: 159-198. 
16. Claing A, Chen W, Miller W E, et al. $\beta$-Arrestin-mediated ADP-ribosylation factor 6 activation and $\beta 2$ adrenergic receptor endocytosis[J]. Journal of Biological Chemistry, 2001, 276(45): 42509-42513.

17. Lee D K, Ferguson S S G, George S R, et al. The fate of the internalized apelin receptor is determined by different isoforms of apelin mediating differential interaction with $\beta$-arrestin[J]. Biochemical and biophysical research communications, 2010, 395(2): 185-189.

18. Liu H, Ding X F, Zhang S G, et al. Effect of esmolol in septic shock patients with tachycardia: a randomized clinical trial[J]. Zhonghua yi xue za zhi, 2019, 99(17): 1317-1322.

19. Wei $\mathrm{C}$, Louis $\mathrm{H}$, Schmitt M, et al. Effects of low doses of esmolol on cardiac and vascular function in experimental septic shock[J]. Critical Care, 2016, 20(1): 407.

20. Quintana-Villamandos B, Delgado-Martos M J, Delgado-Baeza E. Early reversal cardiac with esmolol in hypertensive rats: The role of subcellular organelle phenotype[J]. Pharmacological Reports, 2019, 71(6): 1125-1132.

21. Liu P, Wu Q, Tang Y, et al. The influence of esmolol on septic shock and sepsis: A meta-analysis of randomized controlled studies[J]. The American journal of emergency medicine, 2018, 36(3): 470474.

22. Xinqiang $L$, Weiping $H$, Miaoyun $W$, et al. Esmolol improves clinical outcome and tissue oxygen metabolism in patients with septic shock through controlling heart rate[J]. Zhonghua wei zhong bing ji jiu yi xue, 2015, 27(9): 759-763.

23. Xinqiang $L$, Weiping $H$, Miaoyun $W$, et al. Esmolol improves clinical outcome and tissue oxygen metabolism in patients with septic shock through controlling heart rate[J]. Zhonghua wei zhong bing ji jiu yi xue, 2015, 27(9): 759-763.

24. Philip J L, Xu X, Han M, et al. Regulation of cardiac fibroblast-mediated maladaptive ventricular remodeling by $\beta$-arrestins[J]. PloS one, 2019, 14(7).

25. Schlegel P, Reinkober J, Meinhardt E, et al. G protein-coupled receptor kinase 2 promotes cardiac hypertrophy[J]. PloS one, 2017, 12(7): e0182110.

\section{Figures}




\section{The change of catecholamine levels in plasma}

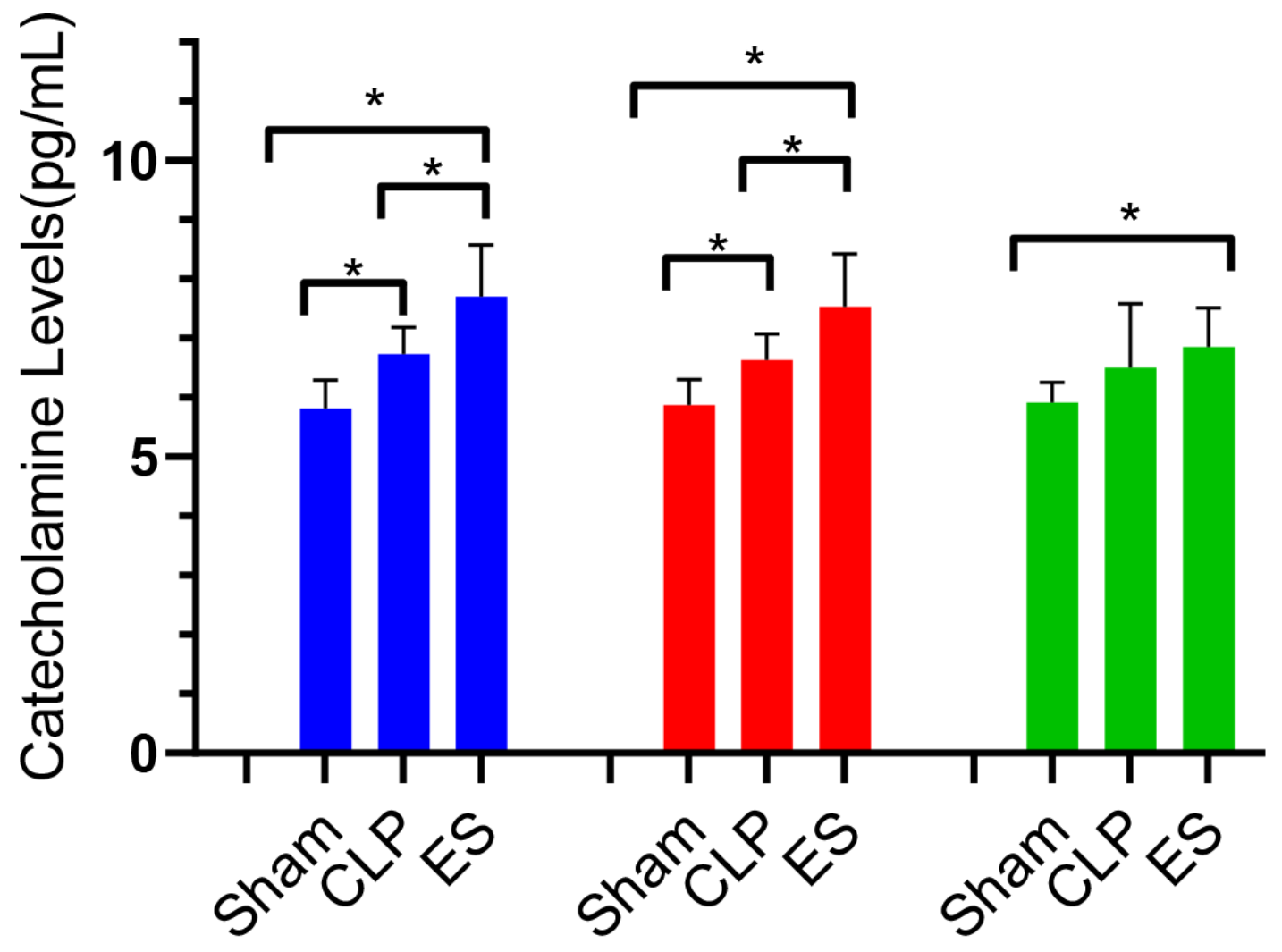

$6 h$

$12 \mathrm{~h}$

$24 \mathrm{~h}$

Figure 1

Assessment of CA levels in plasma as measured by ELISA. Date are expressed as concentration(pg.ml1);black lines indicate medians \pm interquartile range. all groups, $n=8 .{ }^{*} \mathbb{p} \otimes 0.05$.CLP cecal ligation and puncture, ES esmolol 1.5mg.kg-1 .h-1. 
The change of TNF- $\alpha$ levels in plasma

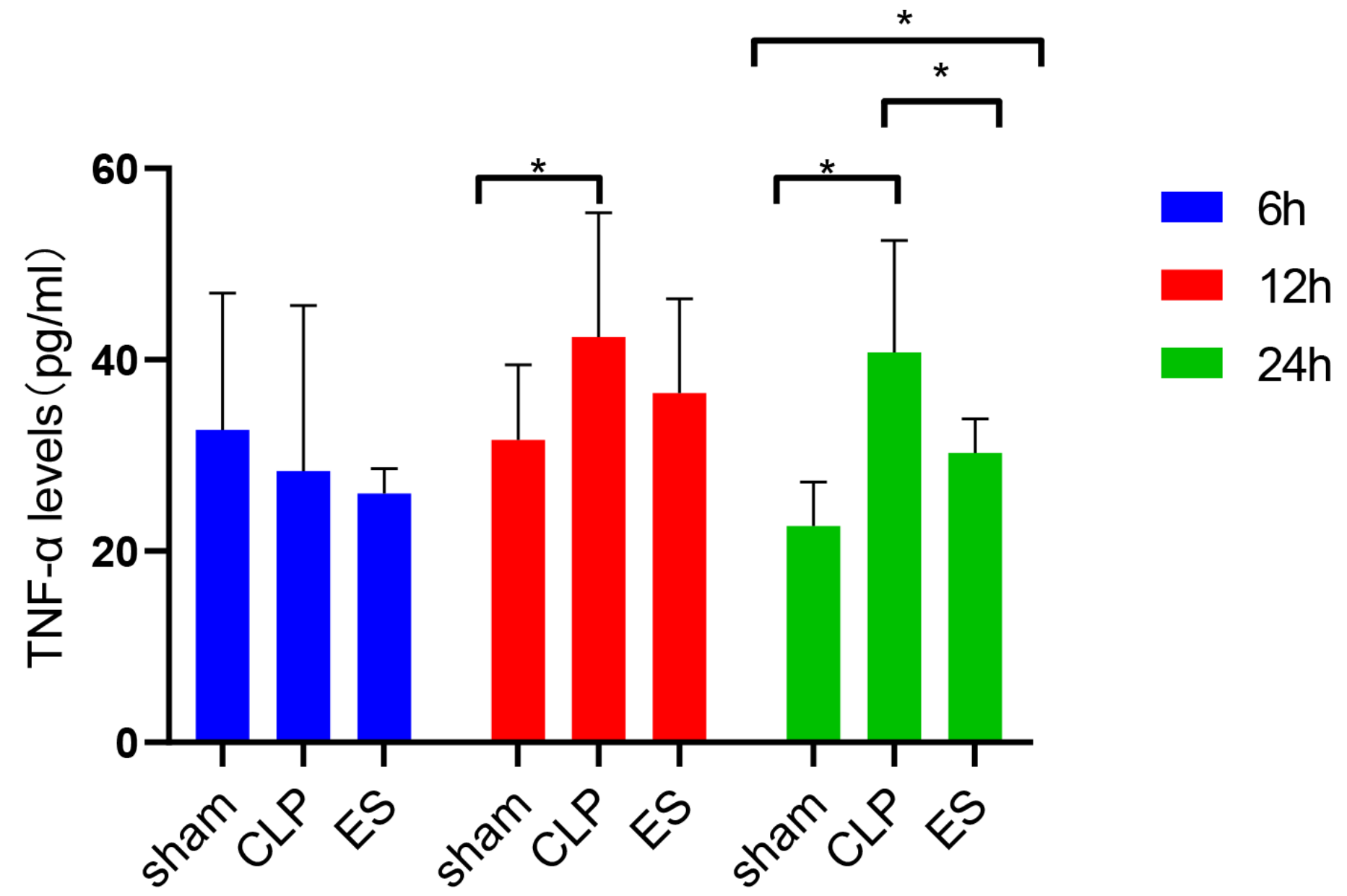

Figure 2

Assessment of TNF-a levels in plasma as measured by ELISA. 
The change of IL-6 levels in plasma

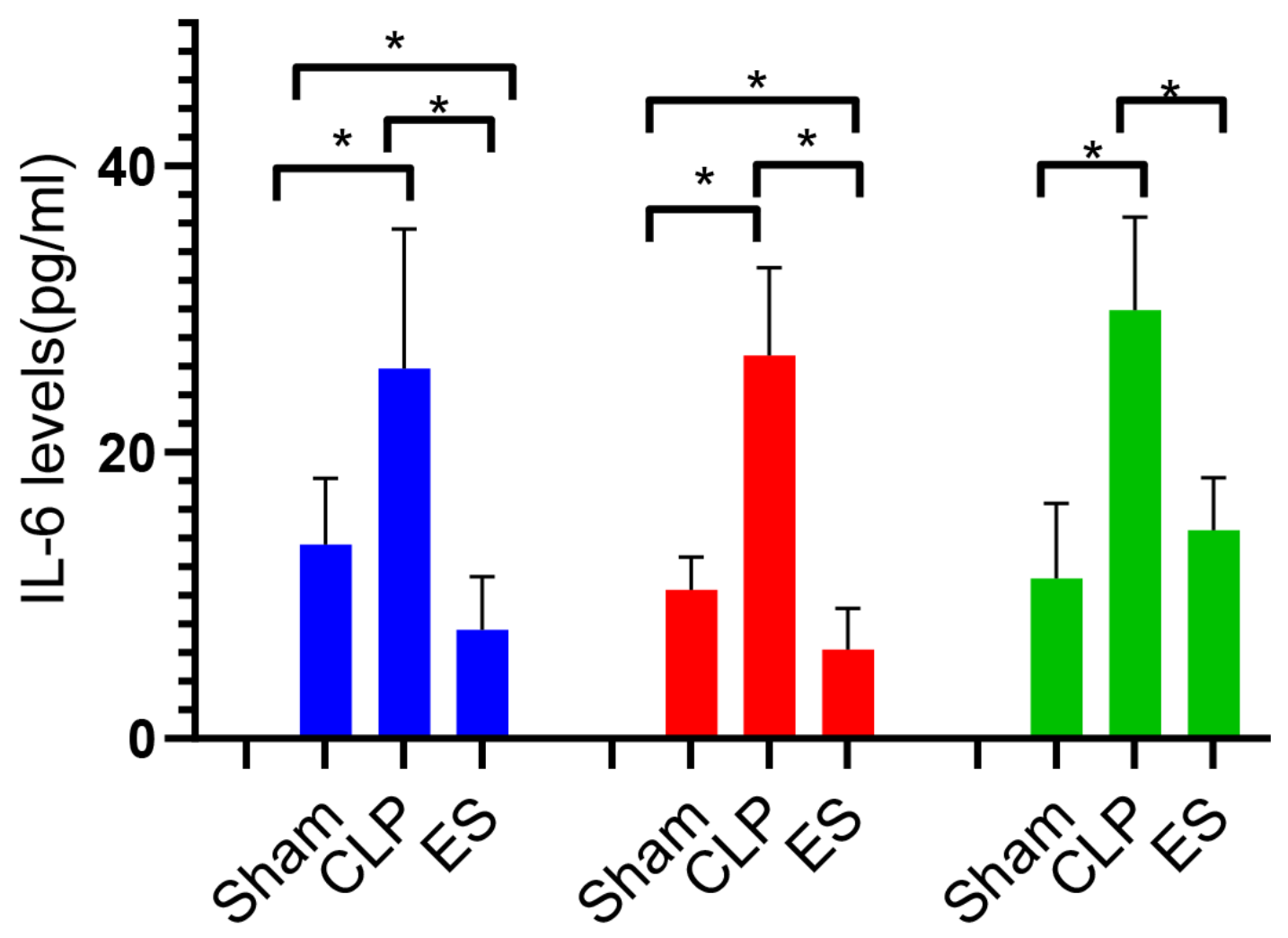

6h

$12 \mathrm{~h}$ $24 h$

Figure 3

Assessment of IL- 6 levels in plasma as measured by ELISA. 
The change of cTNI levels in plasma ( mean \pm SD )

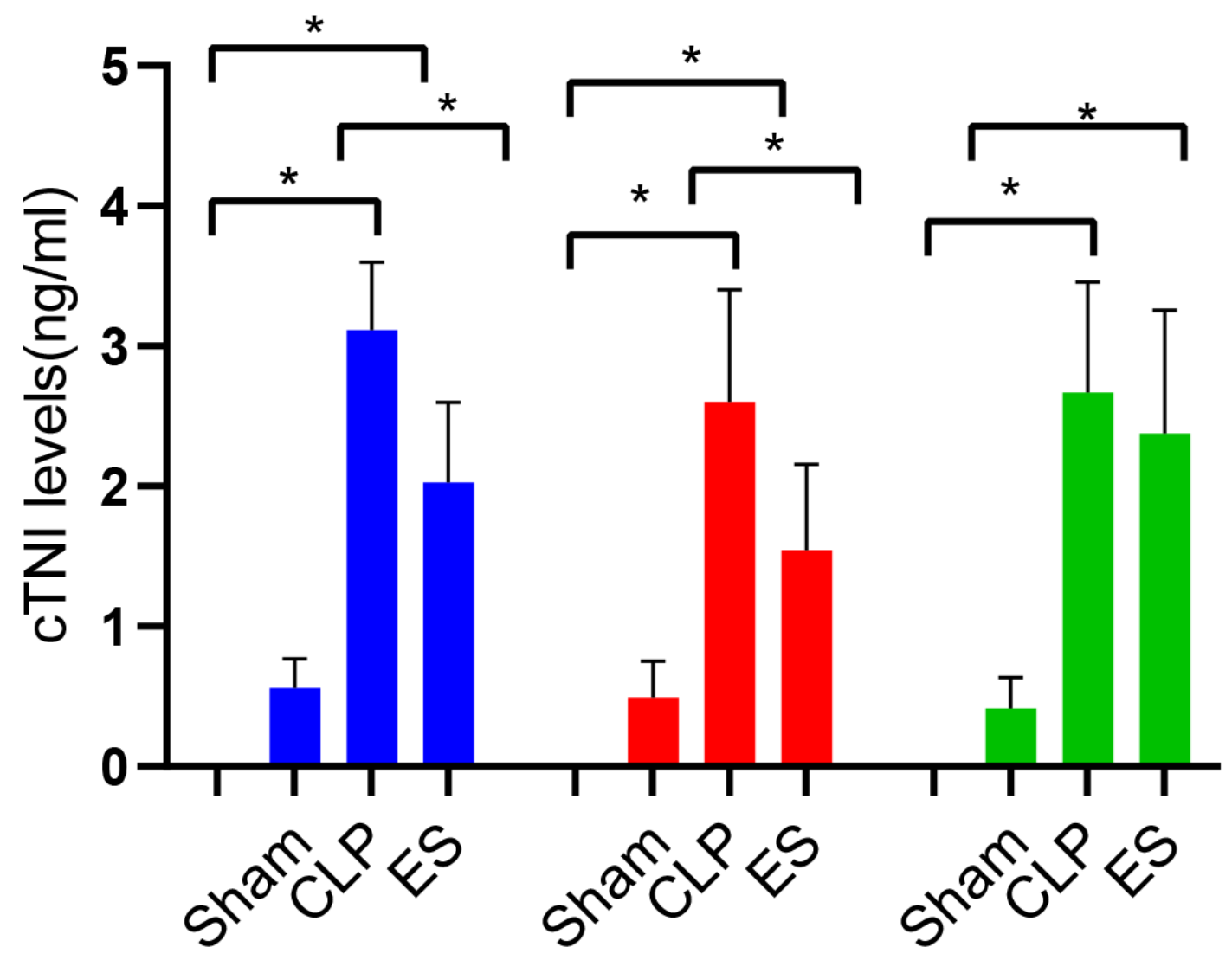

$6 \mathrm{~h}$

$12 \mathrm{~h}$

$24 \mathrm{~h}$

Figure 4

Assessment of cTNI levels in plasma as measured by ELISA. 
The change of $\mathrm{AC}$ levels in plasma

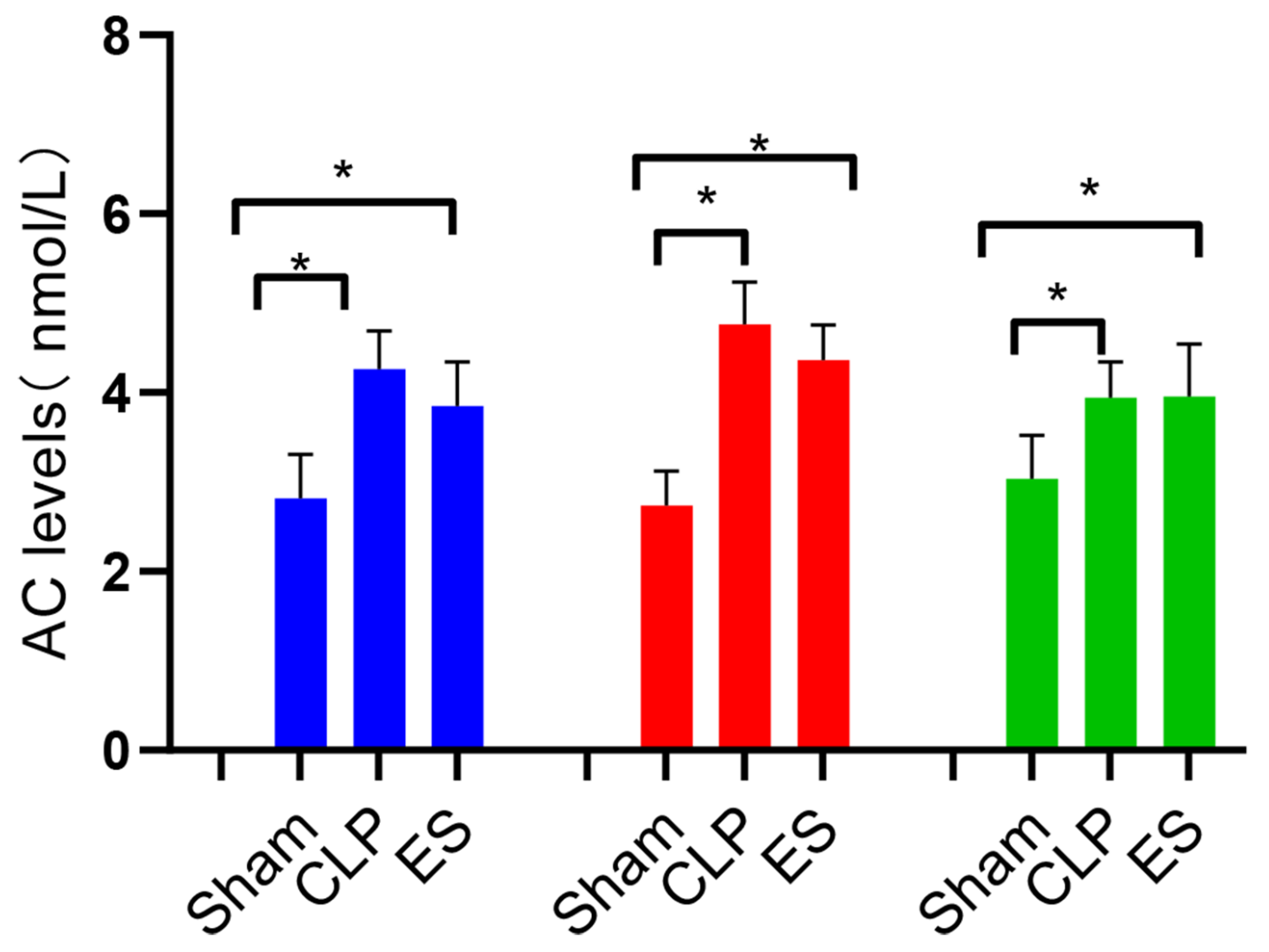

- $6 \mathrm{~h}$

$12 \mathrm{~h}$

$24 \mathrm{~h}$

Figure 5

Assessment of AC levels in plasma as measured by ELISA. 
The change of cAMP levels in plasma

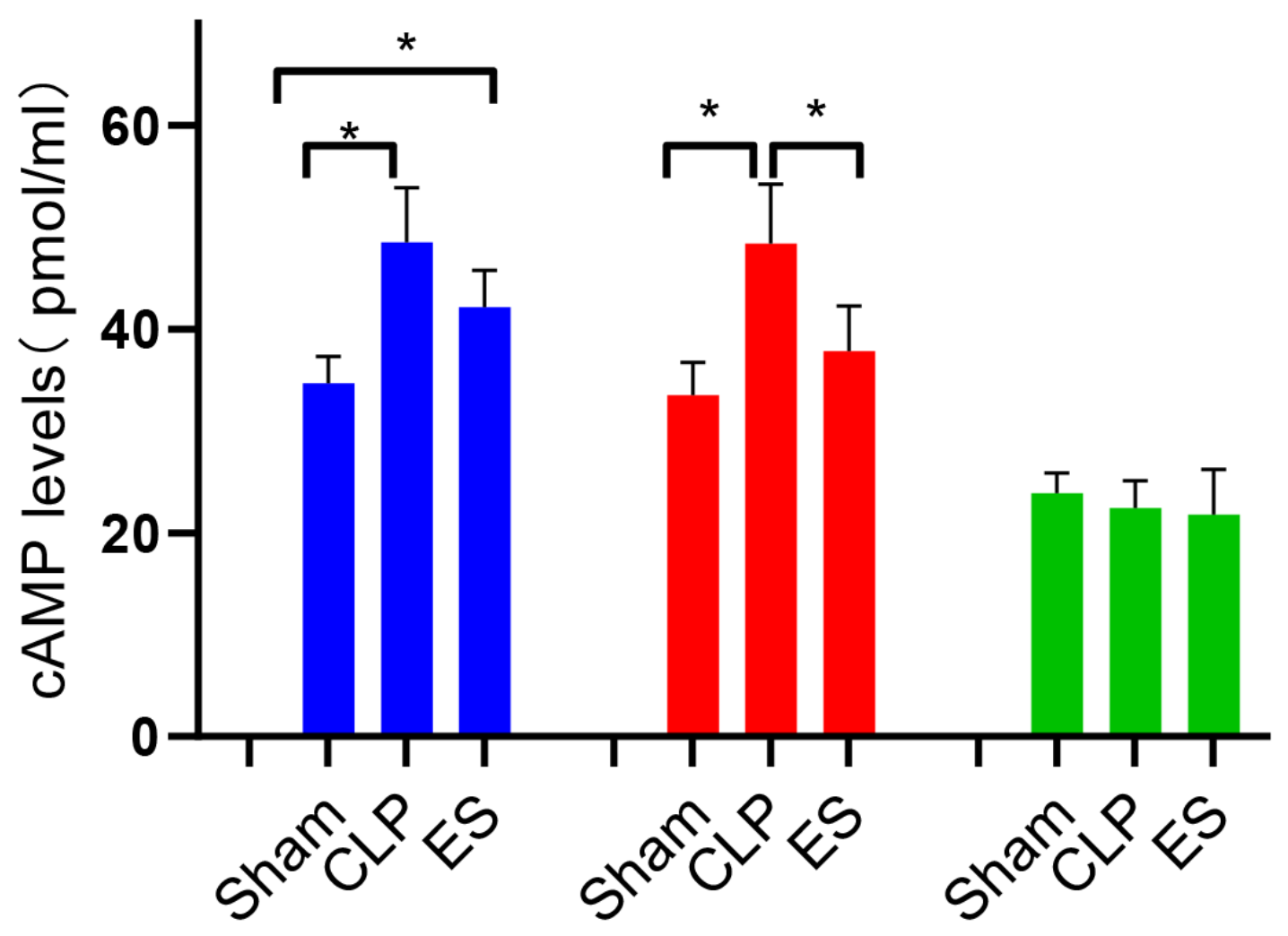

$6 \mathrm{~h}$

$12 \mathrm{~h}$

$24 \mathrm{~h}$

Figure 6

Assessment of cAMP levels in plasma as measured by ELISA. 
The change of PKA levels in plasma

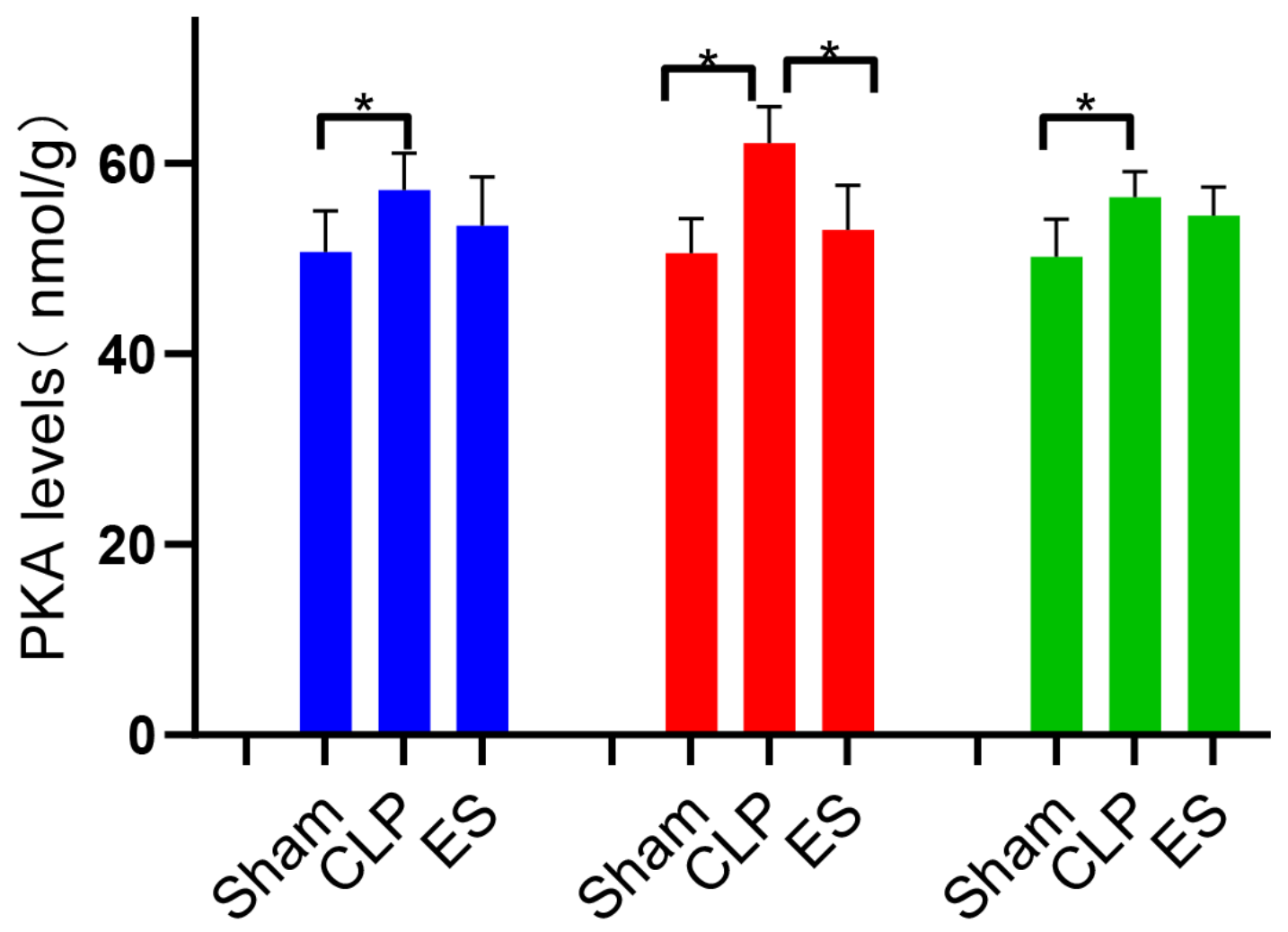

6h

$12 \mathrm{~h}$

$24 \mathrm{~h}$

Figure 7

Assessment of PKA levels in plasma as measured by ELISA. 


\section{$\begin{array}{lllllllllll}\text { S6 } & \text { C6 } & \text { E6 } & \text { S12 } & \text { C12 } & \text { E12 } & \text { S24 } & \text { C24 } & \text { E24 }\end{array}$}
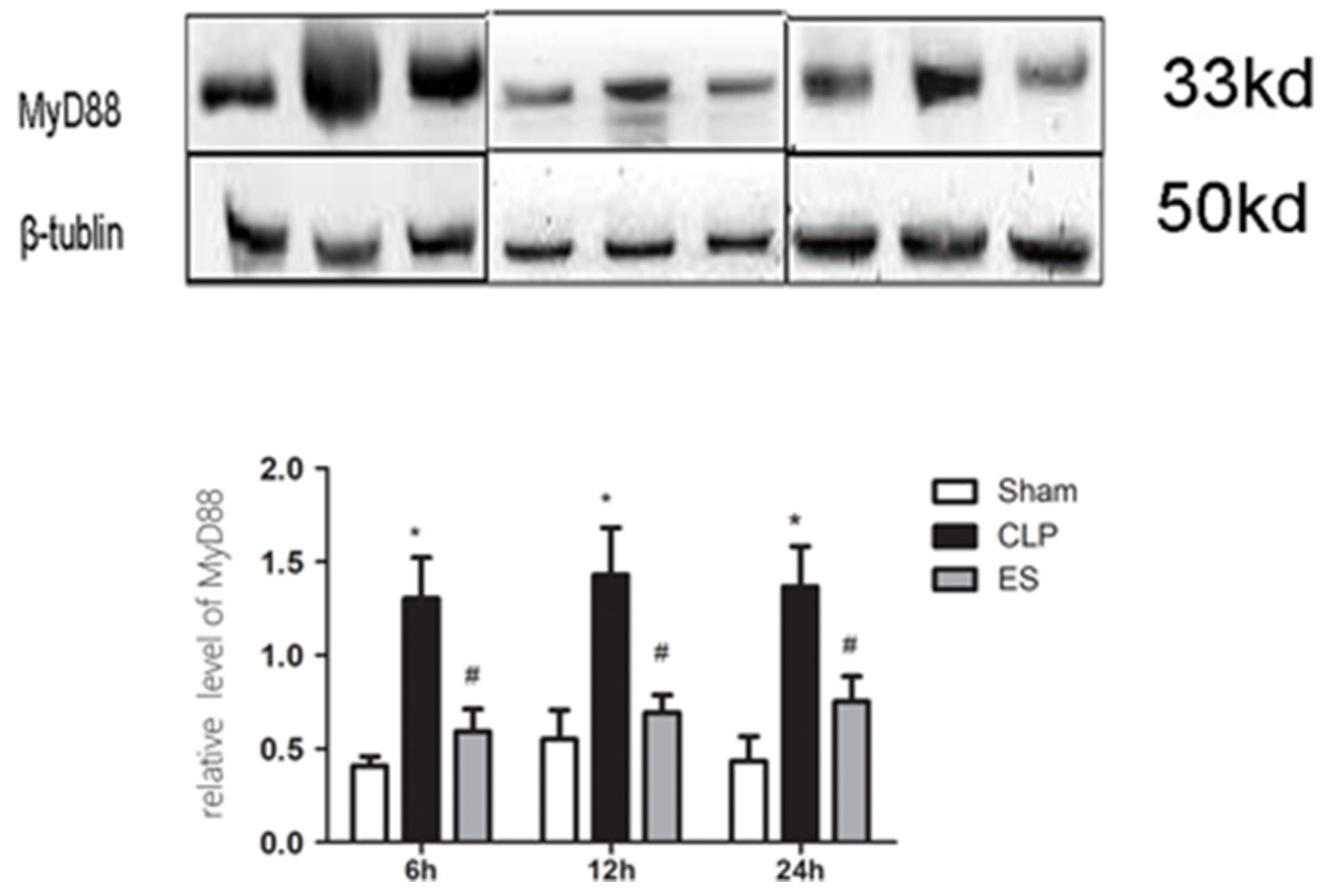

Figure 8

Western blot analysis of protein of MyD88 expression in the myocardium. Proteins were obtained from myocardium $(n=8)$ prepared from all experimental rat groups. A typical western blot is shown above the histogram. Densitometric analysis $(n=8)$ was used to calculate the normalized protein ratio. Data are expressed as median \pm standard deviation range. Upper edges of error bars represent the maximum value in each group. *: Sham VS CLP, $p<0.05$. \#:CLP VS ES, $p<0.05$. CLP: cecal ligation and puncture, ES: esmolol 


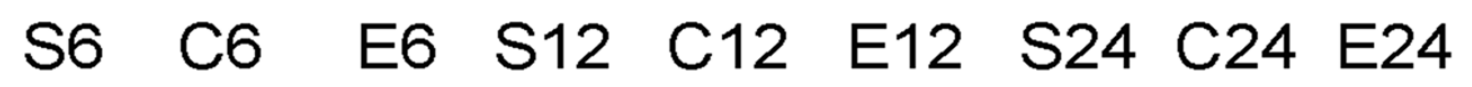
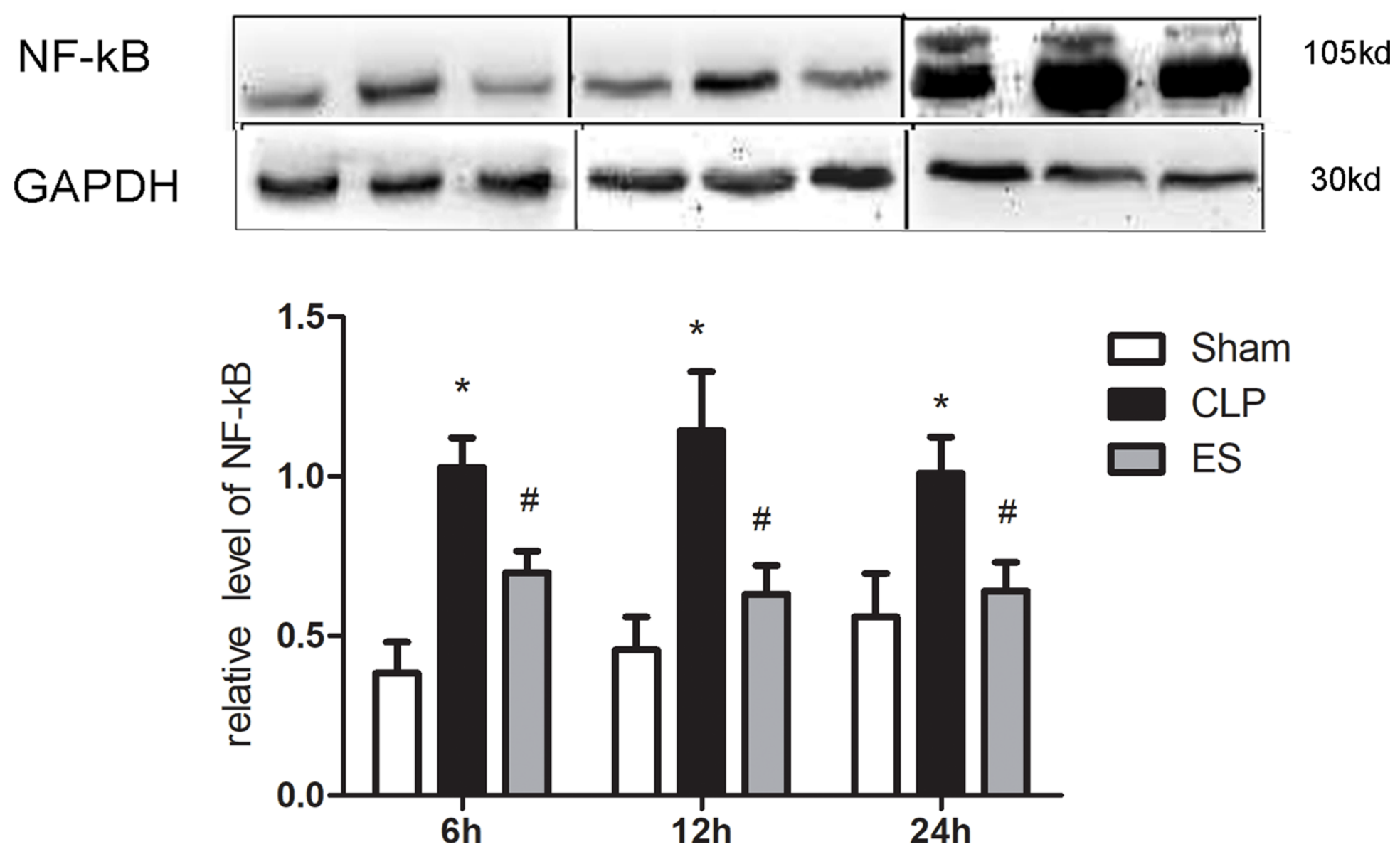

Figure 9

Western blot analysis of protein of Nf-kB expression in the myocardium. 


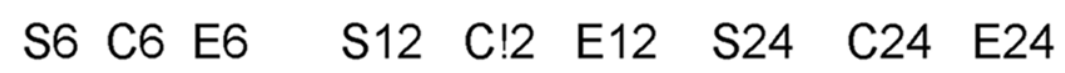
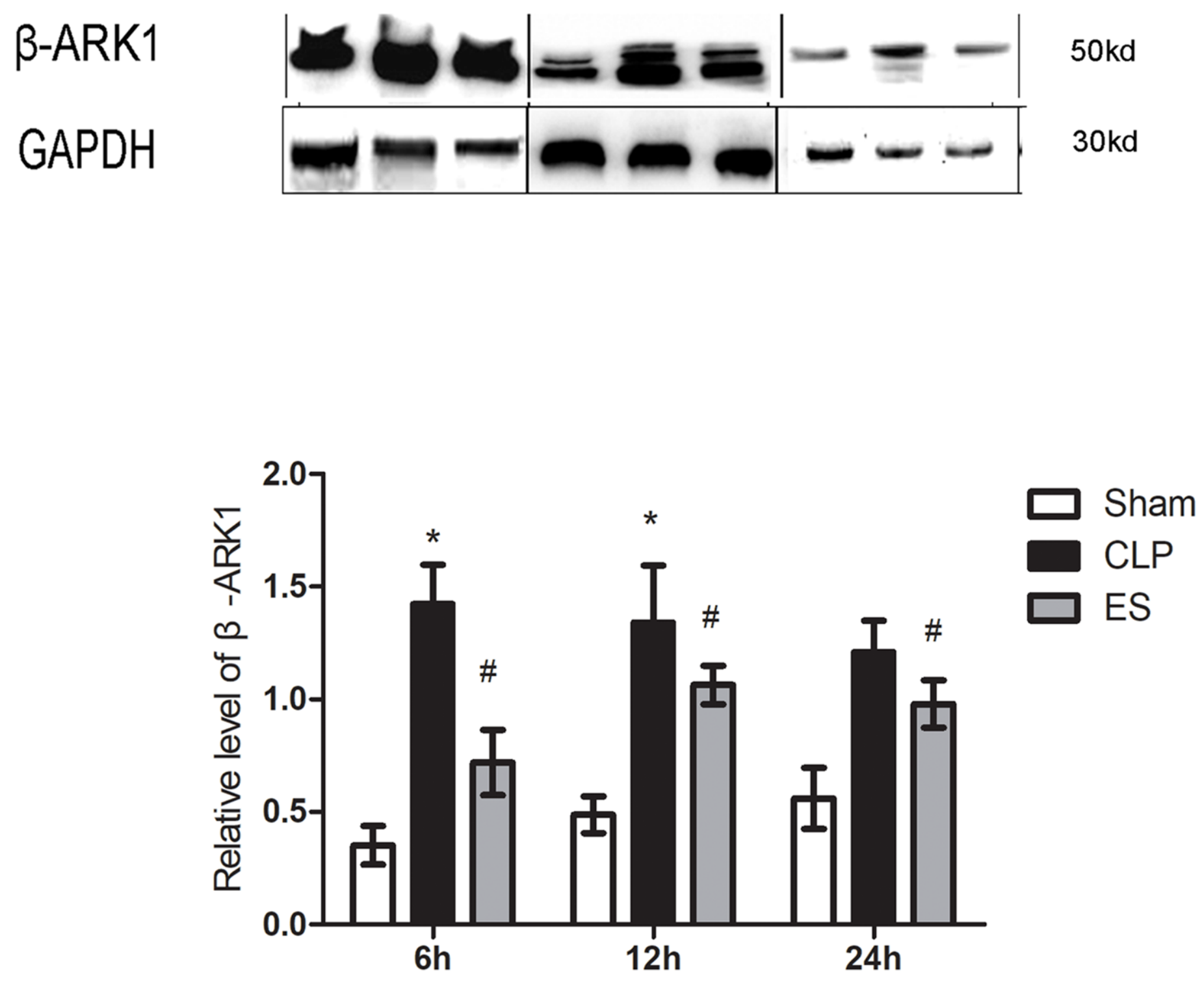

Figure 10

Western blot analysis of protein of $\beta$-ARK1 expression in the myocardium. 

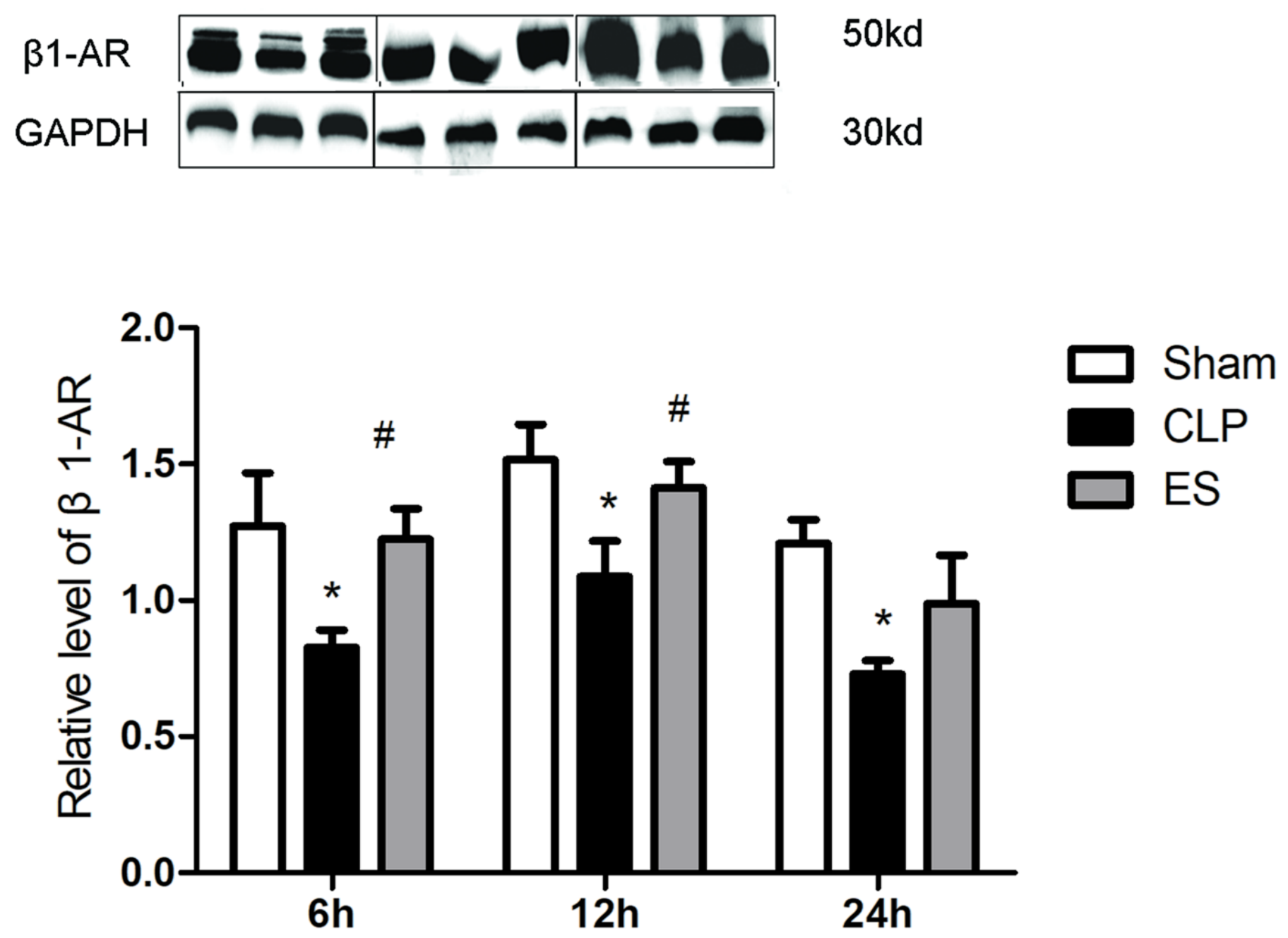

Figure 11

Western blot analysis of protein of $\beta 1$-AR expression in the myocardium. 
$\begin{array}{lllllllllllllll}\text { S6 } & \text { C6 } & \text { E6 } & \text { S12 } & \text { C12 } & \text { E12 } & \text { S24 } & \text { C24 } & \text { E24 }\end{array}$
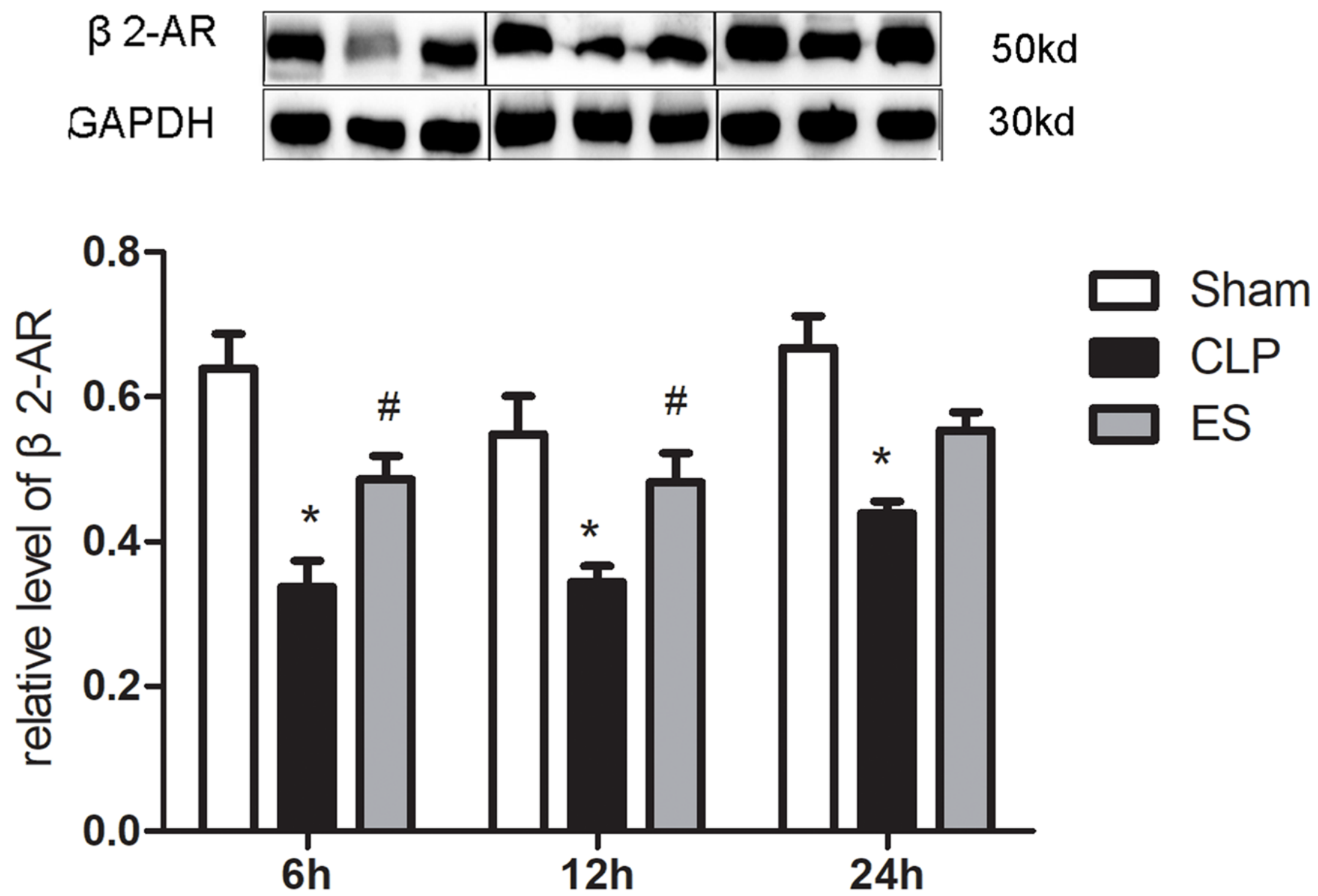

Figure 12

Western blot analysis of protein of $\beta 2$-AR expression in the myocardium. 
S6 C6 E6 S12 C12 E12 S24 C24 E24

$\beta$ arrestin 1 GAPnU

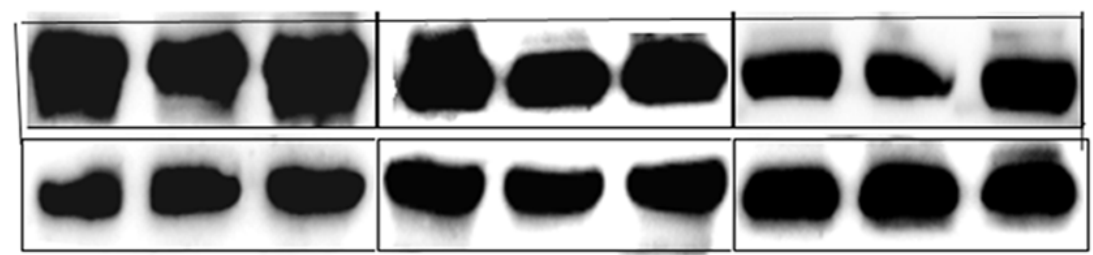

$59 \mathrm{kd}$

$30 \mathrm{kd}$

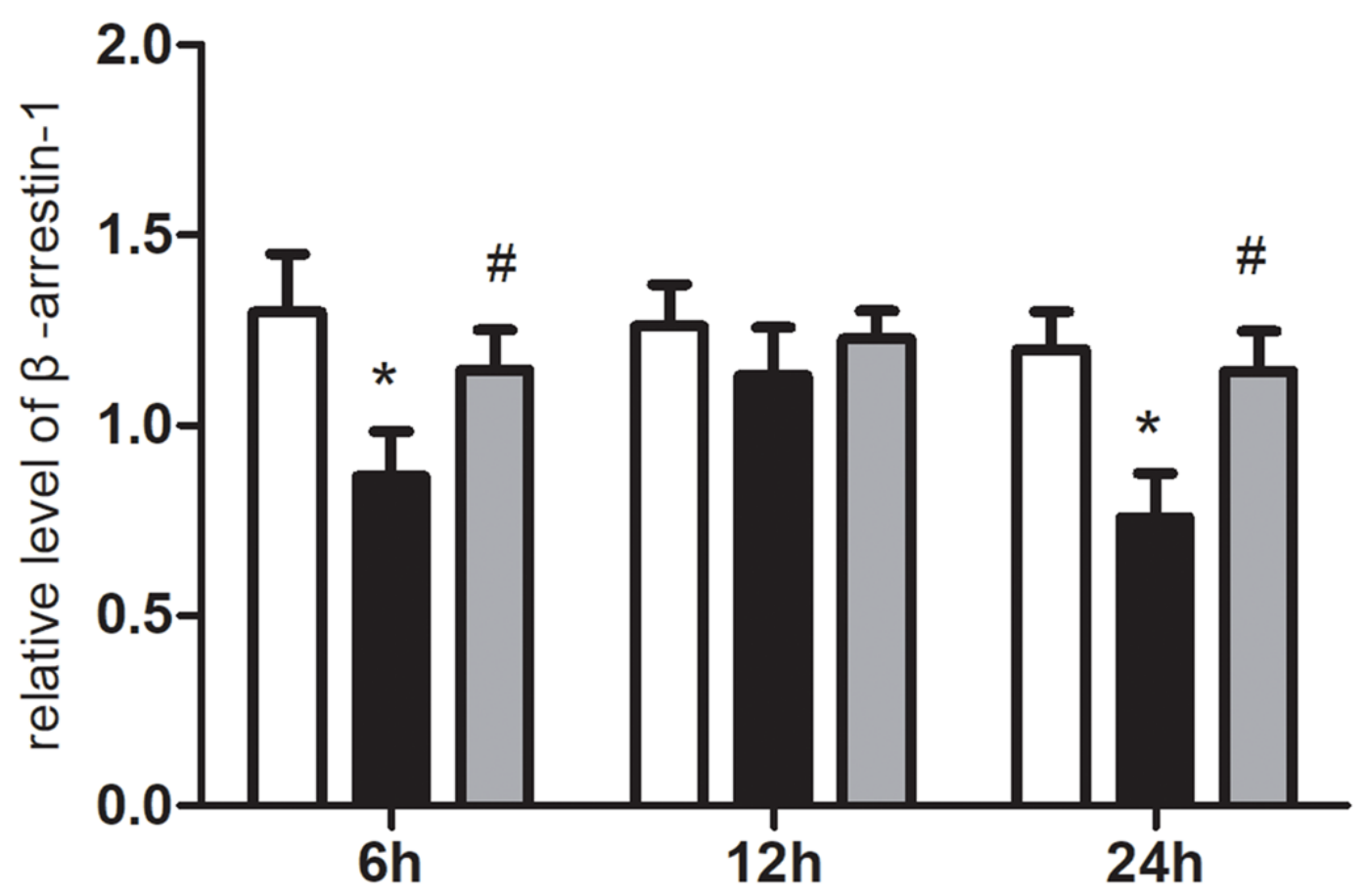

Figure 13

Western blot analysis of protein of $\beta$-arrestin-1 expression in the myocardium. 
$\begin{array}{lllllllll}\text { S6 } & \text { C6 } & \text { E6 } & \text { S12 } & \text { C12 } & \text { E12 } & \text { S24 } & \text { C24 } & \text { E24 }\end{array}$
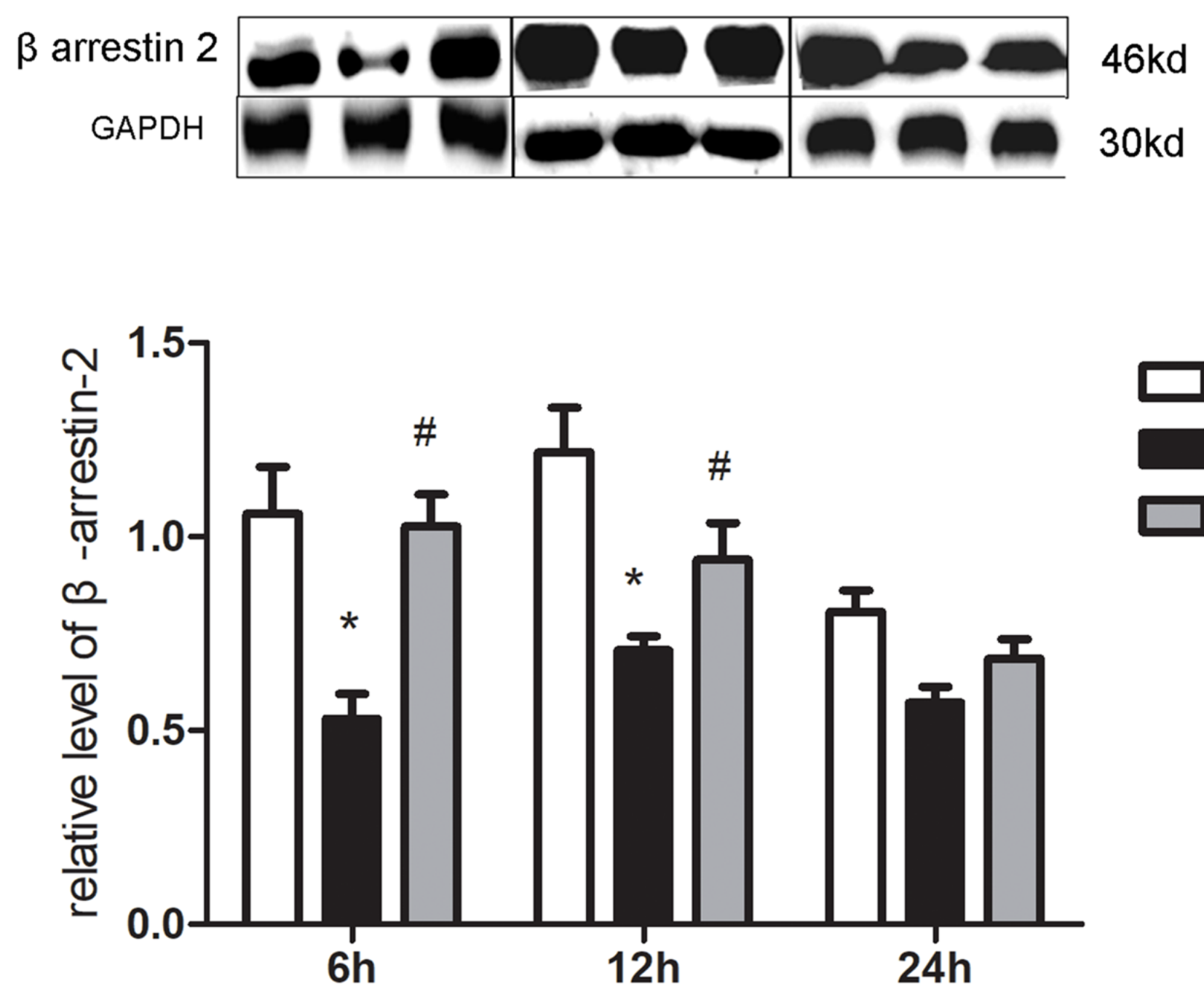

Figure 14

Western blot analysis of protein of $\beta$-arrestin-2 expression in the myocardium. 
Sham

CLP

ES

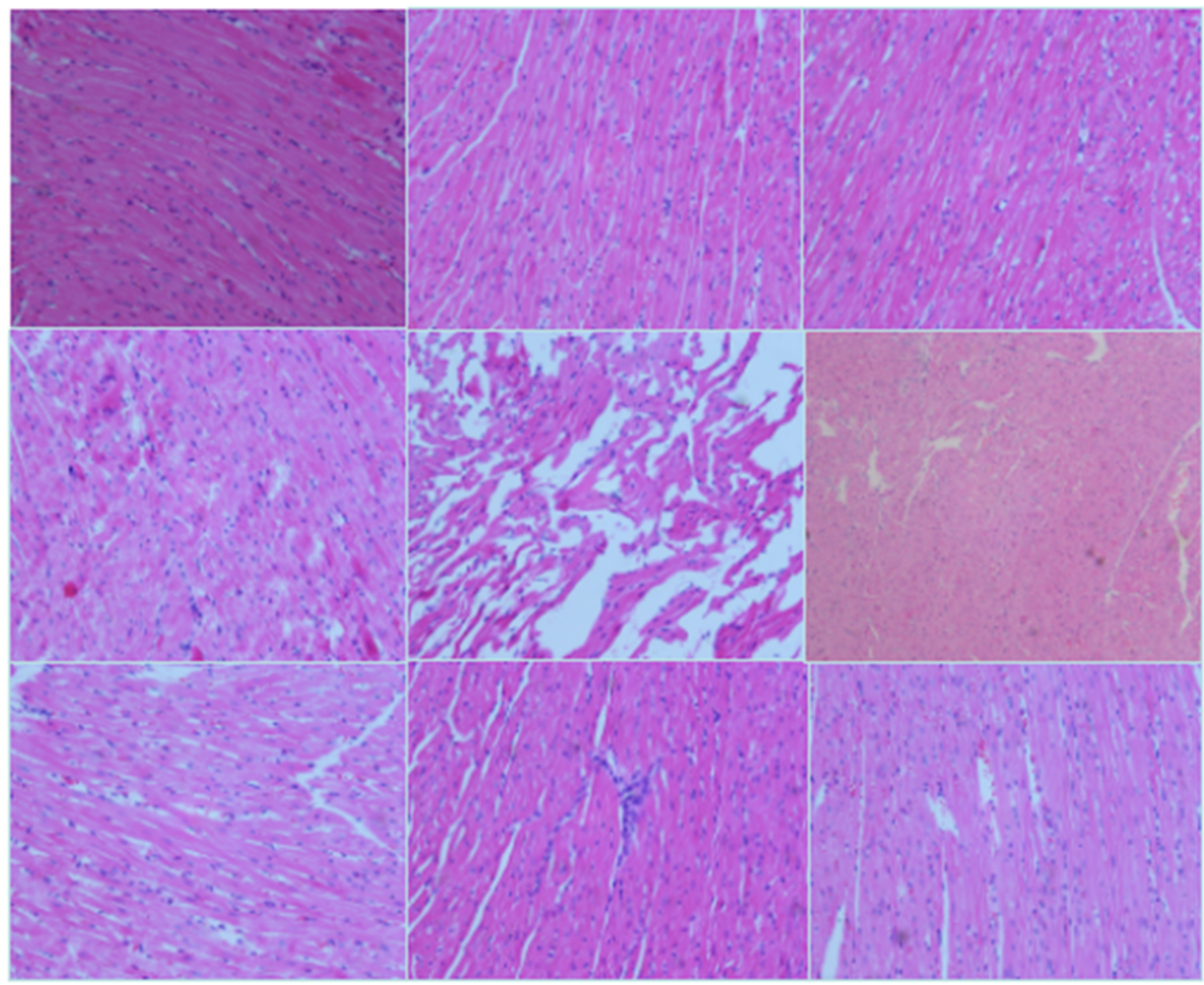

$6 \mathrm{~h}$

$12 \mathrm{~h}$

$24 h$

\section{Figure 15}

The figure shows pathological results, which are sorted chronologically from left to right, and sorted chronologically from top to bottom for different groups. From the figure, we can see different degrees of infiltration of inflammatory cells and myocardial cell damage $(\mathrm{HE} \times 20)$. 
A
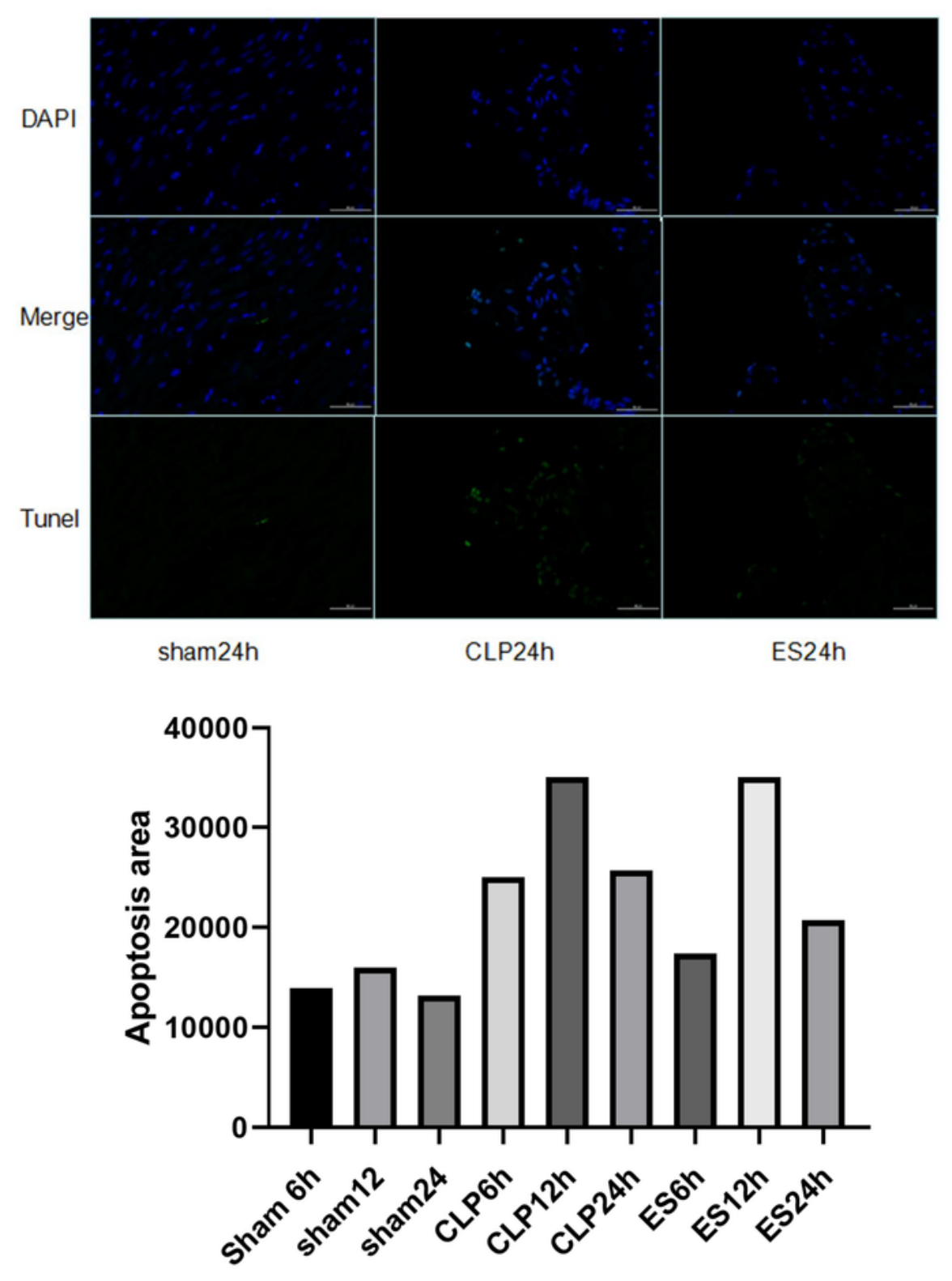

Figure 16

A) This figure shows the immunofluorescence of the $24 \mathrm{~h}$ group, in which cells in the DAPI group that were stained with green fluorescer represent apoptotic cells, and the more staining, the more serious the myocardial cell damage(20x); B) This is a graph of our quantitative analysis using imagine $\mathrm{J}$ statistics 


\section{Survival Analysis}

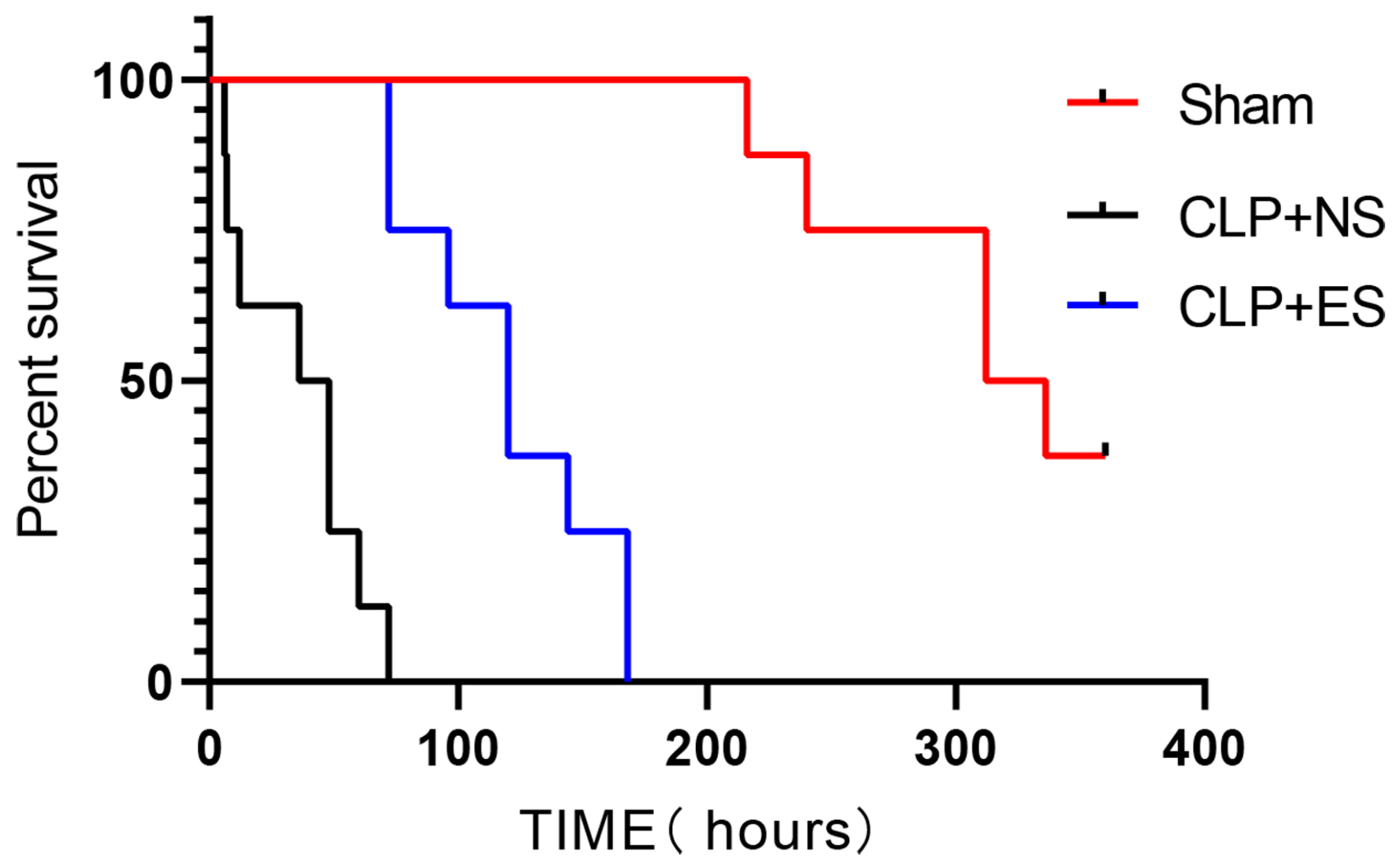

Figure 17

We used the log-rank test for survival analysis to 15 days to compare rat survival curve of Sham group, CLP group, ES group. Using survival analysis at 15 days, the CLP group had the worst outcomes, and the Sham group performed that they had more rats can survive $(p<0.01)$. 